\title{
Multiplierless Approximate 4-point DCT VLSI Architectures for Transform Block Coding
}

\author{
F. M. Bayer* \\ R. J. Cintra ${ }^{\dagger}$ \\ A. Madanayake \\ U. S. Potluri
}

\begin{abstract}
Two multiplierless algorithms are proposed for $4 \times 4$ approximate-DCT for transform coding in digital video. Computational architectures for 1-D/2-D realisations are implemented using Xilinx FPGA devices. CMOS synthesis at the $45 \mathrm{~nm}$ node indicate real-time operation at $1 \mathrm{GHz}$ yielding $4 \times 4$ block rates of $125 \mathrm{MHz}$ at less than $120 \mathrm{~mW}$ of dynamic power consumption.
\end{abstract}

\section{Introduction}

Video and multimedia processing based on signal and image compression such as the high efficiency video coding (HEVC) and H.265 reconfigurable video codecs require 2-D transform block coding for block sizes $N \times N$ where $N \in\{4,8,16,32,64\}[1]$. The transform coding stage requires algorithms for the $N$-point discrete cosine transform (DCT) of types II and IV. The associate transformation matrices are defined, respectively, according to [2]:

$$
\begin{aligned}
{\left[\mathbf{C}_{\mathrm{II}}\right]_{(m, n)} } & =\sqrt{\frac{2}{N}} \cdot \alpha_{m} \cdot \cos \left[\left(m-\frac{1}{2}\right) \cdot \frac{\pi(n-1)}{N}\right], \\
{\left[\mathbf{C}_{\mathrm{IV}}\right]_{(m, n)} } & =\sqrt{\frac{2}{N}} \cdot \cos \left[\left(m-\frac{1}{2}\right) \cdot\left(n-\frac{1}{2}\right) \cdot \frac{\pi}{N}\right],
\end{aligned}
$$

where $m, n=1,2, \ldots, N, \alpha_{1}=1 / \sqrt{2}$, and $\alpha_{m}=1$, for $m>1$.

In this letter, our goal is to propose multiplication-free approximations for the 4-point DCT-II and -IV as well as its fast algorithms. We also aim at VLSI realisations of both 1-D and 2-D versions of the derived approximate transforms, while maintaining at high numerical accuracy and low computational complexity.

${ }^{*}$ F. M. Bayer is with the Departamento de Estatística and Laboratório de Ciências Espaciais de Santa Maria (LACESM), Universidade Federal de Santa Maria, RS, Brazil, E-mail: bayer@ufsm.br

${ }^{\dagger}$ R. J. Cintra is with the Signal Processing Group, Departamento de Estatística, Universidade Federal de Pernambuco, PE, Brazil, E-mail: rjdsc@stat.ufpe.org

${ }^{\ddagger}$ A. Madanayake and U. S. Potluri are with the ECE, The University of Akron, Akron, OH, USA, E-mail: arjuna@uakron.edu 


\section{Optimisation and orthogonalization}

Let $\mathscr{M}_{P}(4)$ be the set of all $4 \times 4$ matrices whose entries are defined over $P=\{-1,0,1\}$. In this set, all matrices represent multiplierless transformations. Our goal is to find matrices in $\mathscr{M}_{P}(4)$ that satisfactorily approximate $\mathbf{C}_{\mathrm{II}}$ and $\mathbf{C}_{\mathrm{IV}}$.

Therefore we propose the following multivariate non-linear optimisation problem over $\mathscr{M}_{P}(4)$

$$
\mathbf{C}_{k}^{*}=\arg \min _{\mathbf{A} \in \mathscr{M}_{P}(4)} \operatorname{error}\left(\mathbf{A}, \mathbf{C}_{k}\right), \quad k \in\{\mathrm{II}, \mathrm{IV}\}
$$

where $\mathbf{C}_{k}^{*}$ are the optimal matrices and $\operatorname{error}(\cdot, \cdot)$ is an error measure between a given candidate matrix and the exact matrices $\mathbf{C}_{\mathrm{II}}$ and $\mathbf{C}_{\mathrm{IV}}$.

Let $h_{i}[n]$ be the discrete signal formed by the $i$ th row of a given matrix $\mathbf{T}$ and the discrete-time Fourier transform (DTFT) of $h_{i}[n]$ be denoted by $H_{i}(\omega ; \mathbf{T})$. As discussed in [3]4], we adopted the total error energy as the error measure. This particular measure is defined as follows:

$$
\varepsilon\left(\mathbf{A}, \mathbf{C}_{k}\right)=\sum_{m=1}^{4} \int_{0}^{\pi}\left|H_{m}(\omega ; \mathbf{A})-H_{m}\left(\omega ; \mathbf{C}_{k}\right)\right|^{2} \mathrm{~d} \omega
$$

for $k \in\{$ II, IV $\}$. In other words, $\varepsilon\left(\mathbf{A}, \mathbf{C}_{k}\right)$ quantifies the sum of the energy error in the DTFT domain—between $\mathbf{A}$ and $\mathbf{C}_{k}$-when the entries of a given matrix row are interpreted as filter coefficients [3,4]. This quantity can be computed numerically by standard quadrature methods [5].

As an additional constraint to (1), we impose that the matrix $\mathbf{A} \cdot \mathbf{A}^{\top}$ must be a diagonal matrix to ensure that orthogonality can be achieved in the obtained approximations [6]. The resulting constrained optimisation problem is algebraically intractable and we resorted to exhaustive computational search.

\section{Proposed 4-point DCT approximations}

By solving 11, we obtained the following new DCT approximations:

$$
\mathbf{C}_{\mathrm{II}}^{*}=\left[\begin{array}{rrrr}
1 & 1 & 1 & 1 \\
1 & 0 & 0 & -1 \\
1 & -1 & -1 & 1 \\
0 & -1 & 1 & 0
\end{array}\right] \text { and } \mathbf{C}_{\mathrm{IV}}^{*}=\left[\begin{array}{rrrr}
1 & 1 & 1 & 0 \\
1 & 0 & -1 & -1 \\
1 & -1 & 0 & 1 \\
0 & -1 & 1 & -1
\end{array}\right] .
$$

Although possessing very low complexity, these matrices are not orthogonal. In several contexts, such as image processing for coding, orthogonality is often a desirable property [2]. Adopting the orthogonalization methods detailed in [6], new orthogonal matrices $\hat{\mathbf{C}}_{\mathrm{II}}$ and $\hat{\mathbf{C}}_{\mathrm{IV}}$ can be derived based on $\mathbf{C}_{\mathrm{II}}^{*}$ and $\mathbf{C}_{\mathrm{IV}}^{*}$, respectively. These orthogonal 


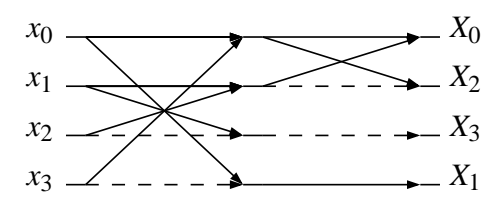

(a) DCT-II approximation

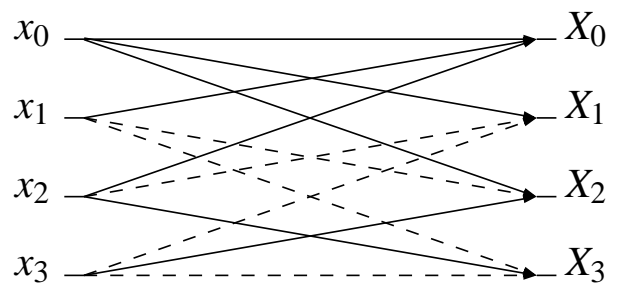

(b) DCT-IV approximation

Figure 1: Signal flow graph for proposed transforms.

matrices are given by:

$$
\hat{\mathbf{C}}_{\mathrm{II}}=\mathbf{D}_{\mathrm{II}} \cdot \mathbf{C}_{\mathrm{II}}^{*} \quad \text { and } \quad \hat{\mathbf{C}}_{\mathrm{IV}}=\mathbf{D}_{\mathrm{IV}} \cdot \mathbf{C}_{\mathrm{IV}}^{*}
$$

where $\mathbf{D}_{\mathrm{II}}=\sqrt{\left[\mathbf{C}_{\mathrm{II}}^{*} \cdot\left(\mathbf{C}_{\mathrm{II}}^{*}\right)^{\top}\right]^{-1}}$ and $\mathbf{D}_{\mathrm{IV}}=\sqrt{\left[\mathbf{C}_{\mathrm{IV}}^{*} \cdot\left(\mathbf{C}_{\mathrm{IV}}^{*}\right)^{\top}\right]^{-1}}$. Explicitly we obtain that

$$
\mathbf{D}_{\mathrm{II}}=\operatorname{diag}\left(\frac{1}{2}, \frac{1}{\sqrt{2}}, \frac{1}{2}, \frac{1}{\sqrt{2}}\right)
$$

and

$$
\mathbf{D}_{\mathrm{IV}}=\frac{1}{\sqrt{3}} \cdot \mathbf{I}_{4},
$$

where $\mathbf{I}_{4}$ is the identity matrix of size 4 . In image compression context the scaling matrices $\mathbf{D}_{\mathbf{1}}$ and $\mathbf{D}_{\mathbf{2}}$ may not introduce any computational overhead, because they can be merged into the quantisation step, as described earlier in [4.7.,9].

The signal flow graph for $\mathbf{C}_{\mathrm{II}}^{*}$ and $\mathbf{C}_{\mathrm{IV}}^{*}$ is shown in Fig.1 We note that the $\mathbf{C}_{\mathrm{II}}^{*}$ and $\mathbf{C}_{\mathrm{IV}}^{*}$ transformations require only 6 and 8 additions, respectively. Multiplications or bit-shifting operations are totally absent. Resulting approximations $\hat{\mathbf{C}}_{\mathrm{II}}$ and $\hat{\mathbf{C}}_{\mathrm{IV}}$ are very close to the respective ideal DCT and offer extremely low complexities. In Table 1 we show the error measure and arithmetic complexity for the proposed transforms, the exact DCT computation [2], and the well-known signed DCT [3]. 
Table 1: Total error energy and arithmetic complexity analysis

\begin{tabular}{lcccc}
\hline \multirow{2}{*}{ Method } & Error & \multicolumn{3}{c}{ Complexity } \\
& Energy & Add. & Mult. & Total \\
\hline Exact 4-point DCT-II [2] & 0.000 & 8 & 4 & 12 \\
4-point Signed DCT-II [3] & 0.957 & 8 & 0 & 8 \\
Proposed $\hat{\mathbf{C}}_{\text {II }}$ & 0.957 & 6 & 0 & 6 \\
\hline Exact 4-point DCT-IV [2] & 0.000 & 12 & 8 & 20 \\
4-point Signed DCT-IV [3] & 2.359 & 10 & 0 & 10 \\
Proposed $\hat{\mathbf{C}}_{\text {IV }}$ & 0.838 & 8 & 0 & 8 \\
\hline
\end{tabular}

Table 2: Resource consumption on Xilinx XC6VSX475T-2FF1156

\begin{tabular}{lcccccc}
\hline $\begin{array}{l}\text { Proposed } \\
\text { Approx. }\end{array}$ & CLB & FF & LUT & Slices & $\begin{array}{c}F_{\max } \\
(\mathrm{MHz})\end{array}$ & $\begin{array}{c}D_{p} \\
(\mathrm{~W})\end{array}$ \\
\hline 1-D DCT-II & 56 & 76 & 92 & 35 & 743.5 & 0.535 \\
1-D DCT-IV & 76 & 132 & 128 & 52 & 735.3 & 0.574 \\
2-D DCT-II & 166 & 408 & 330 & 108 & 704.2 & 0.884 \\
2-D DCT-IV & 210 & 528 & 472 & 148 & 689.2 & 0.921 \\
\hline
\end{tabular}

\section{FPGA prototypes}

The approximate DCTs were realised as an architecture for the 4-point 1-D transforms and the extended to $4 \times 42-\mathrm{D}$ transformation. The inputs were assumed at 8-bit resolution. Rapid prototypes were realised on a Xilinx Virtex-6 field programmable gate array (FPGA) device and tested to ensure correct on-chip functionality. The results concerning the consumption of configurable logic blocks (CLB), flip-flops (FF), look-up tables (LUT), and slices are shown in Table2 The maximum operating frequency $\left(F_{\max }\right)$ and dynamic power consumption $\left(D_{p}\right)$ are also displayed.

The register transfer language (RTL) code corresponding to the FPGA-verified designs were targeted to $45 \mathrm{~nm}$ CMOS standard cell process using Cadence Encounter. The CMOS designs were realised up to synthesis and placeand-route levels leading to the estimated results in Table 3 Area-time complexities AT and AT $^{2}$ were adopted and measured in $\mu \mathrm{m}^{2} \cdot \mathrm{ns}$ and $\mu \mathrm{m}^{2} \cdot \mathrm{ns}^{2}$, respectively.

Table 3: Resource consumption for $45 \mathrm{~nm}$ CMOS

\begin{tabular}{lcccccr}
\hline $\begin{array}{l}\text { Proposed } \\
\text { Approx. }\end{array}$ & $\begin{array}{c}\text { ASIC } \\
\text { Gates }\end{array}$ & $\begin{array}{c}\text { Area } \\
\left(\mu \mathrm{m}^{2}\right)\end{array}$ & $\begin{array}{c}F_{\max } \\
(\mathrm{GHz})\end{array}$ & $\begin{array}{c}D_{p} \\
(\mathrm{~mW})\end{array}$ & AT & $\mathrm{AT}^{2}$ \\
\hline 1-D DCT-II & 849 & 3386.9 & 1.10 & 6.31 & 3160 & 2948 \\
1-D DCT-IV & 1207 & 4870.4 & 1.00 & 8.62 & 4846 & 4822 \\
2-D DCT-II & 7400 & 31217.8 & 0.95 & 59.33 & 7770 & 8159 \\
2-D DCT-IV & 13770 & 59052.5 & 0.94 & 115.66 & 14596 & 15472 \\
\hline
\end{tabular}




\section{Conclusion}

Numerical optimisation methods have lead to 4-point approximations for the DCT-II and DCT-IV. Such matrices are tailored for minimal computational complexity and are adequate for computing realisations linked to coding operations with applications in digital video and multimedia. Fast algorithms were derived and the associate physical realisations do not require VLSI area- and power-intensive multiplier circuits. Both 1-D and 2-D realisations were proposed with FPGA prototypes for architecture validation and CMOS synthesis results at the $45 \mathrm{~nm}$ node. Results indicate real-time blockrate of $125 \mathrm{MHz}$ for processing $4 \times 4$ blocks at $1 \mathrm{GHz}$ clock frequency.

\section{Acknowledgments}

We thank The College of Engineering at UA, CNPq, FACEPE, and FAPERGS for the partial financial support.

\section{References}

[1] G. J. Sullivan, J.-R. Ohm, W.-J. Han, and T. Wiegand, "Overview of the high efficiency video coding (HEVC) standard," IEEE Transactions on Circuits and Systems for Video Technology, vol. 22, pp. 1649-1668, Dec. 2012.

[2] V. Britanak, P. Yip, and K. R. Rao, Discrete Cosine and Sine Transforms. Academic Press, 2007.

[3] T. I. Haweel, "A new square wave transform based on the DCT," Signal Processing, vol. 82, pp. 2309-2319, 2001.

[4] R. J. Cintra and F. M. Bayer, “A DCT approximation for image compression,” IEEE Signal Processing Letters, vol. 18, pp. 579-582, Oct. 2011.

[5] R. Piessens, E. deDoncker-Kapenga, C. Uberhuber, and D. Kahaner, Quadpack: a Subroutine Package for Automatic Integration. Springer-Verlag, 1983.

[6] R. J. Cintra, "An integer approximation method for discrete sinusoidal transforms," Journal of Circuits, Systems, and Signal Processing, vol. 30, no. 6, pp. 1481-1501, 2011.

[7] S. Bouguezel, M. O. Ahmad, and M. N. S. Swamy, "Low-complexity $8 \times 8$ transform for image compression," Electronics Letters, vol. 44, pp. 1249-1250, Sept. 2008.

[8] F. M. Bayer and R. J. Cintra, "DCT-like transform for image compression requires 14 additions only," Electronics Letters, vol. 48, pp. 919-921, July 2012.

[9] S. Bouguezel, M. O. Ahmad, and M. N. S. Swamy, "A low-complexity parametric transform for image compression," in Proceedings of the 2011 IEEE International Symposium on Circuits and Systems, 2011. 